\title{
五甲川菁线粒体苂光探针：光稳定性与激发态 寿命的关系
}

\author{
黄海桥, 龙飒然, 李明乐, 杜健军, 㚞江莉, 彭孝军“ \\ 大连理工大学精细化工国家重点实验室, 大连 116024 \\ *通讯作者, E-mail: pengxj@dlut.edu.cn \\ 收稿日期: 2017-03-24; 接受日期: 2017-04-18; 网络版发表日期: 2017-07-21 \\ 国家自然科学基金(编号：21421005, U1608222)资助项目
}

\begin{abstract}
摘要不同结构的五甲川菁荧光染料常被用于近红外荧光探针, 其灵敏度高, 但光稳定性不理想. 本文合 成了一系列中位引入电子给体对氨基苯或对差基苯的五甲川菁染料, 研究了取代基对光稳定性的影响. 实 验表明, 具有氨基和差基电子给体的染料具有较高的光稳定性. 飞秒瞬态吸收实验证明, 氨基降低了菁染料 分子的激发态寿命, 光稳定性与激发态寿命成负相关, 同时染料能较好地定位于活细胞中的线粒体. 这对设 计高耐光牢度的新型菁染料探针具有重要意义.
\end{abstract}

关键词菁染料, 光稳定性, 激发态寿命, 生物成像, 线粒体

\section{1 引言}

自从1856年Williams ${ }^{[1]}$ 发现“豆粉”蓝菁染料以来, 由于此类染料具有吸收波长范围可调(540 1000 nm)、 摩尔消光系数大、吸收及发射光谱范围可调、合成 容易等优点, 被广泛应用于生物苂光分析 ${ }^{[2,3]}$ 及苂光 成像 ${ }^{[4,5]}$. 虽然目前菁染料应用面较广, 但普遍存在光 化学不稳定的缺点, 特别是近红外菁染料, 如五甲川 (Cy5)、七甲川染料(Cy7)的光稳定性较差, 成为限制 其产业大规模应用的不利因素 ${ }^{[6,7]}$. 因此, 研究和提高 菁染料的光稳定性具有重要的理论和现实意义.

目前所知, 菁类染料光稳定性不足主要是染料分 子被单线态氧氧化导致的, 而单线态氧可以由染料的
激发态与氧反应而形成. 前人研究发现, 通过外加单 线态氧淬灭剂可以提高染料稳定性 ${ }^{[8]}$; 增加染料中间 甲川链的空间位阻可以提高染料光稳定性; 在染料母 体上引入氟、硝基、氰基等吸电子取代基, 可降低共 轭芳环上的 $\pi$ 电子云密度, 起到降低染料与单重态氧 反应活性的作用 ${ }^{[011]}$, 在一定程度上可以提高染料的 光稳定性 ${ }^{[12,13]}$.

本课题组认为, 降低激发态寿命, 可以减少染料 激发态与氧形成单线态氧的几率, 有望提高染料的光 稳定性. 为此, 合成了一系列对称中位苯环取代的五 甲川菁染料, 通过改变苯环上取代基团、调节五甲川 菁染料的激发态寿命来提升光稳定性, 并考察染料在 细胞苂光成像的性能. 研究发现, 与传统研究结论相

引用格式: 黄海桥, 龙飒然, 李明乐, 杜健军, 㚞江莉, 彭孝军. 五甲川菁线粒体苂光探针: 光稳定性与激发态寿命的关系. 中国科学: 化学, 2017, 47: 1035-1043

Huang H, Long S, Li M, Du J, Fan J, Peng X. Pentamethine cyanine located in mitochondria: relationship between photostability and excited state lifetime. Sci Sin Chim, 2017, 47: 1035-1043, doi: 10.1360/N032017-00061 
反, 供电性可使五甲川菁染料光稳定性提高.

\section{2 实验部分}

\section{1 仪器与试剂}

Bruker Avance II $400 \mathrm{Mz}$ Specreometer (400 MHz) 核磁共振仪(Varian公司, 德国), HP1100LC/MSD质谱仪 (HP公司, 美国), Cary 60紫外-可见分光光度计(Agilent 公司, 美国), Cary Eclipse苂光光谱仪(Agilent公司, 美 国), 绝对苂光量子产率仪 (Hamamatsu公司, 日本), FV10-ASW 激光共聚焦荧光显微镜 (奥林巴斯公司, 日本).

MitoTracker Green FM和Hoechst 33342购买于Invitrogen公司(美国). 硅胶(200 300目)购于青岛美高 集团有限公司(中国)用于柱层析. 除2-苯基丙二醛为 Matrix Scientific公司(哥伦比亚)进口, 其他原料为国产 工业级再提纯或国产分析纯试剂, 其中部分原料和溶 剂经过纯化或干燥处理后再使用, 光谱测试中用的是 分析纯溶剂和蒸馏去离子水, 用之前经过干燥重新蒸 馏, 而且溶剂的光学吸收密度在 0.1 以下.

\section{2 中间体及染料的合成}

\subsection{1 中间体的合成}

(1) 中间体1-乙基-2,3,3-三甲基-3H-吲哚啉碘化盐 $\mathbf{I}-$ $\mathbf{1}$ 的合成. 将 2,3,3-三甲基- $3 H$-吲哚啉 $(7.955 \mathrm{~g}, 50 \mathrm{mmol}$ ) 和碘乙烷 $(11.690 \mathrm{~g}, 75 \mathrm{mmol})$ 加入到含 $25 \mathrm{~mL}$ 甲苯的 $100 \mathrm{~mL}$ 单口圆底烧瓶中, 氮气保护. 加热至回流持 续反应, 过夜, 停止反应. 待反应液冷却至室温后, 加入 $50 \mathrm{~mL}$ 乙醚过滤沉淀并用乙醚洗涤滤饼中的杂质. 干 燥后得到 $11.173 \mathrm{~g}$ 粉红色的固体粉末, 粗收率为 $71 \%$. 产品未经进一步纯化, 直接用于下一步反应.

(2) 2-溴丙二醛二苯胺缩合剂的合成 $(\mathbf{I b}-1)^{[14]}$. 取 2,3-二澳-2-丁烯醛酸 $(5.116 \mathrm{~g}, 20 \mathrm{mmol}$ ) 加入到盛有 $34 \mathrm{~mL}$ 无水乙醇的 $100 \mathrm{~mL}$ 两口烧瓶中, 磁力搅拌使其 溶解后, 装上冷凝装置. 另取苯胺 $(3.908 \mathrm{~g}, 42 \mathrm{mmol})$ 溶 于 $18 \mathrm{~mL}$ 无水乙醇中, 常温搅拌下用恒压滴液漏斗将 苯胺溶液加入到两口瓶中, 至少滴加 $10 \mathrm{~min}$, 溶液颜色 很快由无色变成黄绿色, 有大量的气泡冒出, 并且放 出大量的热, 可以观察到冷凝管上有溶剂回流, 加入 完毕后继续在 $40^{\circ} \mathrm{C}$ 的反应条件下搅拌 $1 \mathrm{~h}$, 温度逐渐降 低冷却, 析出金黄色固体, 在强力搅拌下加入 $50 \mathrm{~mL}$ 乙
醚, 过滤后滤饼用乙醇重结晶得到纯的缩合剂, 干燥 后得产品 $5.130 \mathrm{~g}$, 收率为 $85 \%$.

\subsection{2 染料合成}

(1) 染料 II-1 的合成 (1,5-二 (1, ' ' -二乙基-3,3, $3^{\prime}, 3^{\prime}$ 四甲基- $3 H$-吲哚)-3-澳-二碳菁染料, 碘化盐). 取 1-乙 基-2,3,3-三甲基- $3 H$-吲哚啉碘化盐(1.260 g, $4 \mathrm{mmol}$ )和 丙二醛二苯胺盐酸盐缩合剂 $(0.602 \mathrm{~g}, 2 \mathrm{mmol})$, 加入 到 $100 \mathrm{~mL}$ 单口烧瓶中, 然后加入无水醋酸钠 $(0.340 \mathrm{~g}$, $4 \mathrm{mmol}$ )和 $20 \mathrm{~mL}$ 醋酸䣶, 磁力搅拌使其溶解后, 装上冷 凝装置. 氮气保护下加热到 $110^{\circ} \mathrm{C}$, 溶液颜色很快变为 蓝色, 反应 $30 \mathrm{~min}$ 后停止加热, 自然冷却至室温. 用饱 和食盐水洗涤, 分出有机层, 水层用二氯甲烷多次萃 取, 然后与先前的有机层合并, 用无水硫酸钠干燥, 过 滤掉干燥剂, 将溶剂旋转蒸发至蒸干, 残留液用少量二 氯甲烷溶解, 硅胶柱层析分离纯化用无水甲醇/二氯甲 烷 $(1 / 80, v / v)$ 展开剂冲洗, 收集蓝色组分, 蒸干后为带 有金属光泽的蓝色染料, 得到 $0.865 \mathrm{~g}$ 产品, 产率为 $70 \%$.

${ }^{1} \mathrm{H}$ NMR (400 MHz, MeOD): $\delta 8.41$ (d, $J=13.3 \mathrm{~Hz}$, $2 \mathrm{H}, \mathrm{CH}), 7.56$ (d, $J=7.4 \mathrm{~Hz}, 2 \mathrm{H}, \mathrm{ArH}), 7.46$ (t, $J=7.6 \mathrm{~Hz}$, $2 \mathrm{H}, \mathrm{ArH}), 7.40$ (d, $J=7.8 \mathrm{~Hz}, 2 \mathrm{H}, \mathrm{ArH}), 7.33$ (t, $J=7.4 \mathrm{~Hz}$, 2H, ArH), 6.49 (d, J=13.3 Hz, 2H, CH), 4.24 (q, J=7.2 Hz, $\left.4 \mathrm{H}, \mathrm{CH}_{2}\right), 1.76$ (s, 12H, $\left.\mathrm{CH}_{3}\right), 1.45\left(\mathrm{t}, J=7.2 \mathrm{~Hz}, 6 \mathrm{H}, \mathrm{CH}_{3}\right)$. HRMS-ESI: $m / z$ calcd. $\mathrm{M}^{+}$for $\mathrm{C}_{29} \mathrm{H}_{34} \mathrm{BrN}_{2}{ }^{+}, 489.1900$; found, 489.1891. ${ }^{13} \mathrm{C}$ NMR (100 MHz, MeOD) $\delta 175.84$, $151.18,143.08,142.81,129.91,127.04,123.62,116.93$, $112.42,103.30,51.09,40.58,27.45,12.39$.

(2) 染料 II-PHOH 的合成 (1,5-二 (1,1'- 二乙基 -3,3,3', $3^{\prime}$-四甲基-3H-吲哚)-3-(4-羟基苯基)-二碳菁染 料, 碘化盐 $)^{[15,16]}$. 取 III-1 $(0.100 \mathrm{~g}, 0.162 \mathrm{mmol})$ 和对羟 基苯硼酸 $(0.045 \mathrm{~g}, 0.324 \mathrm{mmol})$ 于 $50 \mathrm{~mL}$ 两口圆底烧 瓶中, 依次向其中加入 $\mathrm{K}_{2} \mathrm{CO}_{3}(0.067 \mathrm{~g}, 0.487 \mathrm{mmol})$ 和 四三苯基磷氯化钯 $(0.019 \mathrm{~g}, 0.016 \mathrm{mmol})$, 最后加入 DMF: $\mathrm{H}_{2} \mathrm{O}=1: 1$ 的溶剂 $3 \mathrm{~mL}$, 氮气保护下 $80^{\circ} \mathrm{C}$ 反应 $4 \mathrm{~h}$, 薄层色谱(TLC)监控, II-1基本反应完全, 停止反应. 冷却至室温, 先用水洗涤产物, 再用二氯甲烷萃取 $(20 \mathrm{~mL} \times 3)$, 无水 $\mathrm{Na}_{2} \mathrm{SO}_{4}$ 干燥, 旋转蒸发除去溶剂, 硅胶 柱层析分离纯化用无水甲醇/二氯甲烷 $(1 / 60, v / v)$ 展开 剂冲洗, 收集蓝色组分, 蒸干后为带有金属光泽的蓝 色染料, 得 $0.035 \mathrm{~g}$ 产品, 产率为 $34 \%$.

${ }^{1} \mathrm{H}$ NMR $\left(400 \mathrm{MHz}, \mathrm{CD}_{2} \mathrm{Cl}_{2}\right) \delta 7.96(\mathrm{~d}, J=13.9 \mathrm{~Hz}$, $2 \mathrm{H}, \mathrm{CH}), 7.42$ (d, J=7.4 Hz, 2H, ArH), 7.39 (m, 2H, ArH), 
7.31 (d, $J=8.4 \mathrm{~Hz}, 2 \mathrm{H}, \mathrm{ArH}), 7.25$ (d, $J=7.5 \mathrm{~Hz}, 2 \mathrm{H}, \mathrm{ArH})$, 7.09 (t, $J=7.9 \mathrm{~Hz}, 4 \mathrm{H}, \mathrm{ArH}), 5.85(\mathrm{~d}, J=13.9 \mathrm{~Hz}, 2 \mathrm{H}$, $\mathrm{CH}), 3.79$ (q, J=7.2 Hz, 4H, $\mathrm{CH}_{2}$ ), 1.75 (s, $12 \mathrm{H}, \mathrm{CH}_{3}$ ), 1.22 (t, $J=7.2 \mathrm{~Hz}, 6 \mathrm{H}, \mathrm{CH}_{3}$ ). HRMS-ESI: $m / z$ calcd. $\mathrm{M}^{+}$ for $\mathrm{C}_{35} \mathrm{H}_{39} \mathrm{~N}_{2} \mathrm{O}^{+}, 503.3057$; found, 503.3054. ${ }^{13} \mathrm{C}$ NMR $\left(100 \mathrm{MHz}, \mathrm{CD}_{2} \mathrm{Cl}_{2}\right) \delta 172.85,153.64,142.06,141.62$, $131.23,129.11,125.72,122.74,117.21,110.90,101.96$, 49.73, 39.66, 30.09, 28.21, 12.31 .

(3) 染料 II-PHNH 2 的合成 (1,5-二 (1,1'-二乙基 -3,3,3',3'-四甲基-3H-吲哚)-3-(4-氨基苯基) -二碳菁 染料, 碘化盐). 取 II-1 ( $0.100 \mathrm{~g}, 0.162 \mathrm{mmol})$ 和对氨基 苯硼酸盐酸盐 $(0.056 \mathrm{~g}, 0.324 \mathrm{mmol})$ 于 $50 \mathrm{~mL}$ 两口圆底 烧瓶中, 依次向其中加入 $\mathrm{K}_{2} \mathrm{CO}_{3}(0.067 \mathrm{~g}, 0.487 \mathrm{mmol})$ 和四三苯基磷氯化钯 $(0.019 \mathrm{~g}, 0.016 \mathrm{mmol})$, 最后加入 DMF: $\mathrm{H}_{2} \mathrm{O}=1: 1$ 的溶剂 $3 \mathrm{~mL}$, 氮气保护下 $80^{\circ} \mathrm{C}$ 反应 $4 \mathrm{~h}$, TLC监控, II-1基本反应完全, 停止反应. 冷却至室温, 先用水洗涤产物, 再用二氯甲烷萃取 $(20 \mathrm{~mL} \times 3)$, 无 水 $\mathrm{Na}_{2} \mathrm{SO}_{4}$ 干燥, 旋转蒸发除去溶剂, 硅胶柱层析分离 纯化用无水甲醇/二氯甲烷 $(1 / 60, v / v)$ 展开剂冲洗, 收 集蓝色组分, 蒸干后为带有金属光泽的蓝色染料, 得 $0.031 \mathrm{~g}$ 产品, 产率为 $30 \%$.

${ }^{1} \mathrm{H}$ NMR $\left(400 \mathrm{MHz}, \mathrm{CD}_{2} \mathrm{Cl}_{2}\right) \delta 7.99(\mathrm{~d}, J=13.9 \mathrm{~Hz}$, 2H, CH), 7.44 (d, $J=7.4 \mathrm{~Hz}, 2 \mathrm{H}, \mathrm{ArH}), 7.39$ (t, $J=7.7 \mathrm{~Hz}$, $2 \mathrm{H}, \mathrm{ArH}), 7.27$ (t, $J=7.4 \mathrm{~Hz}, 2 \mathrm{H}, \mathrm{ArH}), 7.11$ (d, $J=7.8 \mathrm{~Hz}$, 2H, ArH), 7.05 (d, $J=7.7 \mathrm{~Hz}, 2 \mathrm{H}, \mathrm{ArH}), 6.91(\mathrm{~d}, J=7.7 \mathrm{~Hz}$, $2 \mathrm{H}, \mathrm{ArH}), 5.89$ (d, $J=13.8 \mathrm{~Hz}, 2 \mathrm{H}, \mathrm{CH}), 3.83$ (q, $J=6.9 \mathrm{~Hz}$, $\left.4 \mathrm{H}, \mathrm{CH}_{2}\right), 1.77\left(\mathrm{~s}, 12 \mathrm{H}, \mathrm{CH}_{3}\right), 1.25(\mathrm{t}, J=7.4 \mathrm{~Hz}, 8 \mathrm{H}$, $\mathrm{CH}_{3}, \mathrm{NH}_{2}$ ). HRMS-ESI: $\mathrm{m} / z$ calcd. $\mathrm{M}^{+}$for $\mathrm{C}_{35} \mathrm{H}_{40} \mathrm{~N}_{3}{ }^{+}$, 502.3217; found, 502.3219 .

${ }^{13} \mathrm{C}$ NMR $\left(100 \mathrm{MHz}, \mathrm{CD}_{2} \mathrm{Cl}_{2}\right) \delta 172.74,153.69$, $147.38,141.95,141.57,131.10,128.98,125.60,122.72$, $115.87,110.79,101.78,49.68,39.56,28.10,12.25$.

(4) 染料II-PH的合成 $\left(1,5\right.$-二(1, $1^{\prime}$-二乙基- $3,3,3^{\prime}, 3^{\prime}$ 四甲基-3H-吲哚)-3-(苯基)-二碳菁染料, 碘化盐). 取1乙基-2,3,3-三甲基- $3 H$-吲哚啉碘化盐(1.260 g, $4 \mathrm{mmol}$ ) 和2-苯基丙二醛 $(0.296 \mathrm{~g}, 2 \mathrm{mmol})$, 加入到 $100 \mathrm{~mL}$ 单口 烧瓶中, 加入 $20 \mathrm{~mL}$ 冰醋酸, 然后加入 3 滴吡啶, 磁力 摚拌使其溶解后, 装上冷凝装置. 氮气保护下加热到 $80^{\circ} \mathrm{C}$, 溶液颜色很快变为蓝色, 反应 $30 \mathrm{~min}$ 后停止加热, 自然冷却至室温. 停止反应, 冷却至室温, 先用水洗 涤产物, 再用二氯甲烷萃取 $(20 \mathrm{~mL} \times 3)$, 无水 $\mathrm{Na}_{2} \mathrm{SO}_{4}$ 干
燥, 旋转蒸发除去溶剂, 硅胶柱层析分离纯化用无水 甲醇/二氯甲烷 $(1 / 60, v / v)$ 展开剂冲洗, 收集蓝色组分, 蒸干后为带有金属光泽的蓝色染料, 得到 $0.707 \mathrm{~g}$ 产品, 产率为 $57 \%$.

${ }^{1} \mathrm{H}$ NMR (400 MHz, MeOD) $\delta 8.37$ (d, $J=14.0 \mathrm{~Hz}$, $2 \mathrm{H}, \mathrm{CH}), 7.60$ (t, $J=7.5 \mathrm{~Hz}, 2 \mathrm{H}, \mathrm{ArH}), 7.51(\mathrm{~d}, J=7.4 \mathrm{~Hz}$, 2H, ArH), 7.39 (t, $J=7.7 \mathrm{~Hz}, 2 \mathrm{H}, \mathrm{ArH}), 7.32$ (d, $J=7.2 \mathrm{~Hz}$, 2H, ArH), $7.26(\mathrm{~m}, 4 \mathrm{H}, \mathrm{ArH}), 5.76(\mathrm{~d}, J=14.0 \mathrm{~Hz}, 2 \mathrm{H}$, $\mathrm{CH}), 3.82$ (q, $\left.J=7.2 \mathrm{~Hz}, 4 \mathrm{H}, \mathrm{CH}_{2}\right), 1.78\left(\mathrm{~s}, 12 \mathrm{H}, \mathrm{CH}_{3}\right)$, $1.16\left(\mathrm{t}, J=7.2 \mathrm{~Hz}, 6 \mathrm{H}, \mathrm{CH}_{3}\right)$. HRMS-ESI: $m / z$ calcd. $\mathrm{M}^{+}$ for $\mathrm{C}_{35} \mathrm{H}_{39} \mathrm{~N}_{2}{ }^{+}$, 487.3108; found, 487.3129. ${ }^{13} \mathrm{C}$ NMR $(100 \mathrm{MHz}, \mathrm{MeOD}) \delta 174.31,154.44,142.95,142.77$, $131.36,130.36,129.76,129.28,126.45,123.51,111.82$, $102.21,50.61,39.93,27.78,12.19$.

\section{3 探针分子光谱测试方法}

染料在不同溶剂中的吸收与发射光谱以及苂光 量子产率的测试. 通过图1和2合成的染料经过真空干 燥后, 在万分之一天平上精确称量, 溶于 $5 \mathrm{~mL}$ 二甲基 亚砜(DMSO)中, 配成浓度为 $1.0 \mathrm{mmol} / \mathrm{L}$ 的DMSO染料 母液, 在冰箱中 $\left(0 \sim 4^{\circ} \mathrm{C}\right)$ 保存备用. 测试准备时, 用微量 进样器量取 $3.0 \mu \mathrm{L}$ 的探针母液, 并将其溶于含有 $3 \mathrm{~mL}$ 测试体系溶液的石英比色典中, 混合均匀, 得到测试 体系中探针的浓度为 $1.0 \mu \mathrm{mol} / \mathrm{L}$, 用于吸收光谱和荧光 发射光谱的测试. 染料的荧光量子产率 $\Phi_{\mathrm{f}}$ 通过绝对荧 光量子产率仪测得.

\section{4 光稳定性实验}

用于染料光稳定性测试的装置示意图如图3所示. 染料样品配成 $5.0 \mu \mathrm{mol} / \mathrm{L}$ 的溶液, 放在石英皿中密封. 将 $500 \mathrm{~W}$ 碘铇灯放在样品池正前方 $30 \mathrm{~cm}$ 处. 中间放置

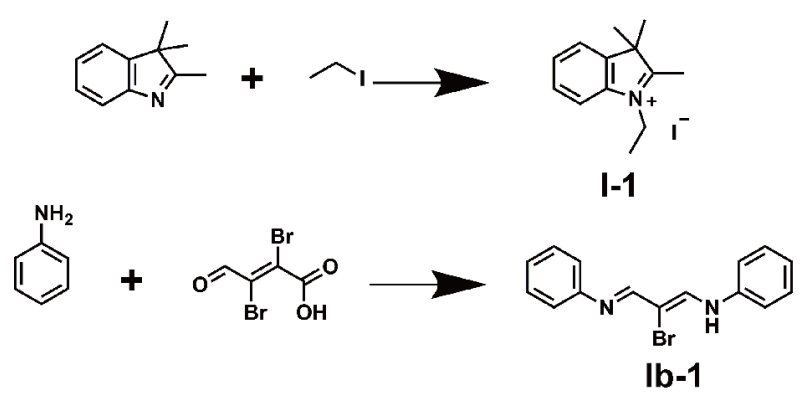

图 1 中间体的合成路线 

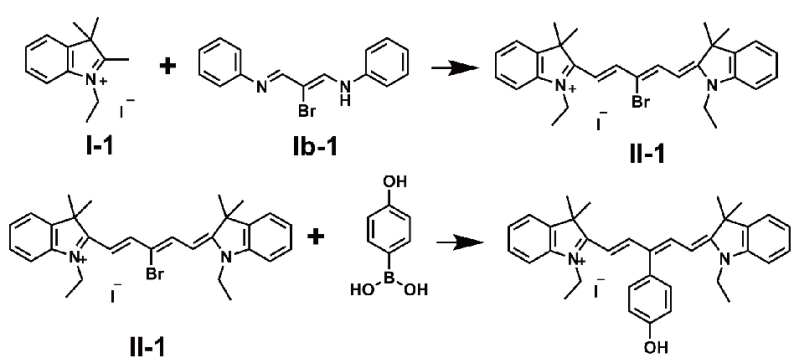

II-1

II-PHOH

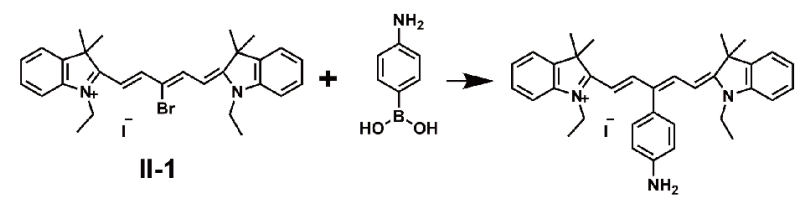

II-PHNH

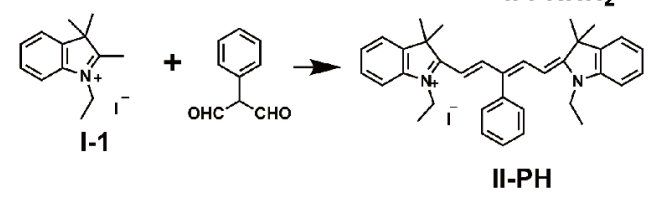

图 2 染料的合成路线

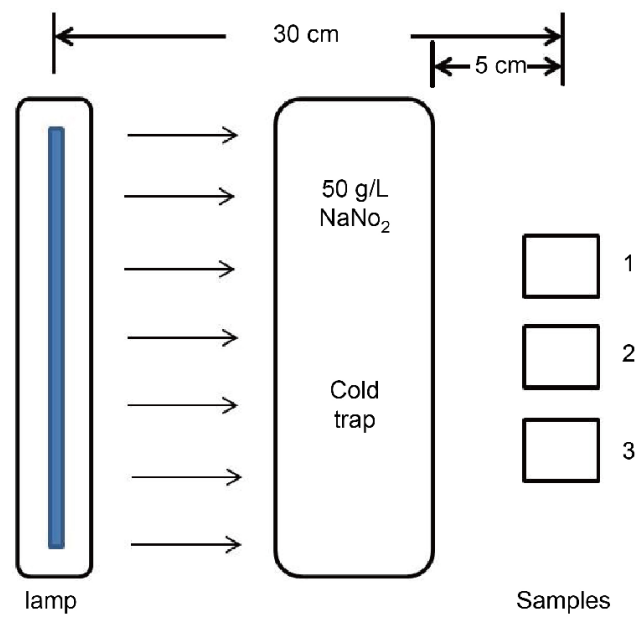

图 3 光稳定性实验装置示意图(网络版彩图)

一个大的玻璃展开缸(厚为 $5 \mathrm{~cm}$ ), 样品距离玻璃展开 缸为 $5 \mathrm{~cm}$, 里面盛有 $50 \mathrm{~g} / \mathrm{L}$ 的亚硝酸钠水溶液 ${ }^{[17]}$, 用作 滤光器, 滤去 $400 \mathrm{~nm}$ 以下的紫外光, 同时可以起到冷 阱的作用, 隔断碘铇灯的强热. 在紫外可见分光光度 仪上测出样品照射前后的吸光度值, 根据吸光度值随 光照时间的下降速度来比较样品的光稳定性.

\section{5 染料的瞬态吸收光谱测试}

飞秒瞬态吸收光谱是在自行搭建的飞秒宽带㬌 浦探测仪器测试得到. 激发波长为 $600 \mathrm{~nm}$, 脉冲宽度 1038
为 50 fs. 进行百浦探测实验前, 调整染料溶液的浓度, 使其在 $1 \mathrm{~mm}$ 厚的石英样品池中, $600 \mathrm{~nm}$ 处吸光度为 $0.12 \sim 0.15$ OD.

\section{6 细胞罦育和复染实验}

将可传代的人乳腺癌细胞系(MCF-7)接种在共聚 焦皿上, 在适量的培养液中培养 $\left(37^{\circ} \mathrm{C}, 5 \% \mathrm{CO}_{2}\right) 24 \mathrm{~h}$ 后, 用磷酸盐缓冲溶液(PBS)冲洗共聚焦血 3 次, 移除缓冲 溶液, 然后加入新鲜培养基培养细胞以供后续实验.

将接种好的MCF-7细胞与 $0.2 \mu \mathrm{mol} / \mathrm{L}$ 的MitoTracker Green FM以及 $1.0 \mu \mathrm{mol} / \mathrm{L}$ 的染料依照上述的染色操作 孵育 $20 \mathrm{~min}$, 再将 $2.0 \mu \mathrm{g} / \mathrm{mL}$ 的核酸染料Hoechst 33342 加入上述细胞继续孵育 $10 \mathrm{~min}$, 移除培养基, 用PBS缓 冲溶液将细胞洗涤 3 次, 向共聚焦血中加入新鲜的培 养基, 然后使用放大倍数为 60 倍的油镜作为物镜, 分 别拍摄其明场和荧光图像.

\section{3 结果与讨论}

\section{1 染料的光谱性能}

染料在不同溶剂中的吸收和发射性质见表 1 , 染 料II-PH、II-PHOH和II-PHNH ${ }_{2}$ 的最大吸收和发射峰 (均在 650 和 $670 \mathrm{~nm}$ ), 说明中位取代基对其波长影响较 小, 并且各染料在不同溶剂中的紫外吸收和发射光谱 的变化规律一致. 在醇类等质子性溶剂中, 随溶剂极性 增大, 如从甲醇、乙醇到水, 染料的紫外-可见吸收和 发射光谱发生蓝移, 表现为负溶剂化效应. 如图4所示, 染料 II-PHNH 2 的最大吸收峰在乙醇、甲醇和水(极 性: 乙醇 4.3 , 甲醇 6.6 , 水 10.2) 中分别为 $647 、 643$ 和 $639 \mathrm{~nm}$.

从表 1 中染料的荧光量子产率可以看出, 3 个染料 在二氯甲烷 $(\mathrm{DCM})$ 、乙腈等有机溶剂中的苂光量子 产率均比水中高, 这是由于染料与水分子之间相互作 用, 染料的非辐射衰减过程增加所致. 对于五甲川菁 染料而言, 吲哚环之间亚甲基链的顺反异构化是非辐 射衰减的主要方式, 染料与水分子的作用使得染料分 子的扭曲角增大, 破坏了分子的共平面性, 导致非辐 射衰减过程的增加, 造成能量损耗, 从而使染料在水 中的苂光有比较明显的猝灭 ${ }^{[18]}$.

同时可以看出, II-PHOH $(0.075, \mathrm{DCM})$ 和 II-PHNH $_{2}(0.023$, DCM $)$ 的苂光量子产率明显小于 II-PH $(0.157$, DCM $)$, 说明中位苯环取代对菁染料激发 

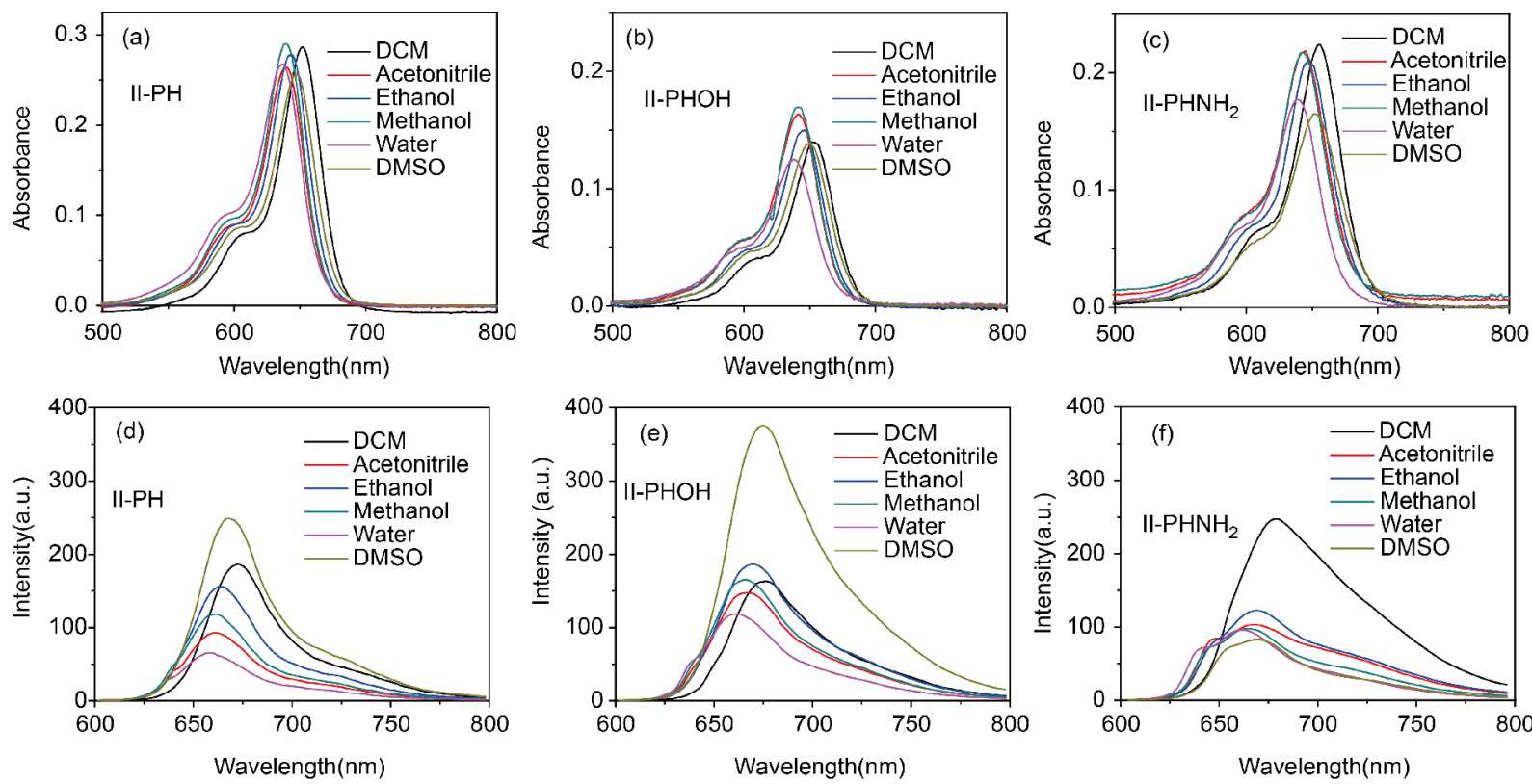

图 4 染料的吸收和发射图谱(网络版彩图)

表 1 染料在不同溶剂中的光谱性能

\begin{tabular}{|c|c|c|c|c|c|c|}
\hline 染料 & 溶剂 ${ }^{a)}$ & $\lambda_{\mathrm{abs}}{ }^{\mathrm{b})}(\mathrm{nm})$ & $\lambda_{\mathrm{em}}{ }^{\mathrm{c}}(\mathrm{nm})$ & $\begin{array}{c}\text { 斯托克斯位 } \\
\text { 移 }(\mathrm{nm})\end{array}$ & $\Phi_{\mathrm{f}}{ }^{\mathrm{d})}$ & $\begin{array}{c}\varepsilon^{\mathrm{e})}\left(10^{5} \mathrm{~mol}^{-1}\right. \\
\left.\mathrm{cm}^{-1} \mathrm{~L}\right)\end{array}$ \\
\hline \multirow{6}{*}{ II-PH } & 二氯甲烷 & 652 & 672 & 20 & 0.157 & 2.87 \\
\hline & 乙腈 & 639 & 661 & 22 & 0.061 & 2.65 \\
\hline & 乙醇 & 644 & 663 & 19 & 0.096 & 2.79 \\
\hline & 甲醇 & 639 & 662 & 23 & 0.061 & 2.91 \\
\hline & 水 & 637 & 658 & 22 & 0.038 & 2.68 \\
\hline & 二甲基亚砜 & 647 & 668 & 21 & 0.157 & 2.57 \\
\hline \multirow{6}{*}{ II-PHOH } & 二氯甲烷 & 652 & 676 & 24 & 0.075 & 1.40 \\
\hline & 乙腈 & 642 & 667 & 25 & 0.043 & 1.64 \\
\hline & 乙醇 & 646 & 670 & 24 & 0.053 & 1.50 \\
\hline & 甲醇 & 641 & 667 & 26 & 0.037 & 1.70 \\
\hline & 水 & 638 & 661 & 23 & 0.028 & 1.26 \\
\hline & 二甲基亚砜 & 650 & 675 & 25 & 0.129 & 1.39 \\
\hline \multirow{6}{*}{ II-PHNH } & 二氯甲烷 & 656 & 679 & 23 & 0.023 & 2.24 \\
\hline & 乙腈 & 645 & 668 & 23 & 0.012 & 2.19 \\
\hline & 乙醇 & 647 & 669 & 22 & 0.011 & 2.11 \\
\hline & 甲醇 & 643 & 664 & 21 & 0.009 & 2.18 \\
\hline & 水 & 639 & 661 & 22 & 0.008 & 1.77 \\
\hline & 二甲基亚砜 & 652 & 669 & 17 & 0.009 & 1.65 \\
\hline
\end{tabular}

a) 所有光谱测试温度为 $25^{\circ} \mathrm{C}$; b) 最大吸收波长; c)最大发射波长; d) 测试浓度为 $\left.1.0 \mu \mathrm{mol} / \mathrm{L} ; \mathrm{e}\right)$ 摩尔消光系数 
态基本无影响, 但是当中位苯环对位引入羟基和氨基 后对菁染料甲川链母体结构有明显的荧光猝灭.

\section{2 染料光稳定性测试}

本文研究了 3 个不同取代基团的菁染料在水中的 光降解性质. 染料的光稳定测试结果如图5 所示, 染料 在水中, 经碘铇灯照射 $4 \mathrm{~h}$, 各染料的剩余吸光率顺序是:

II-PH $(4 \%)<$ II-PHOH $(12 \%)<$ II-PHNH $2(60 \%)$, 计算染 料的光降解速率常数分别是: II-PH (0.78)>II-PHOH $(0.54)>\mathbf{I I}-\mathbf{P H N H} \mathbf{H}_{2}(0.12)$. 结果表明, 染料 II-PHNH 2 具 有最高的光稳定性, 其次是II-PHOH、II-PH. 但由于 氨基是供电基团, 与传统吸电基团有利于光稳定性的 预测相反. 因此, 对于该分子体系而言, 可能存在其他 影响光稳定性的因素.

\section{3 染料瞬态吸收测试}

菁染料的光降解主要是由于共轭链受到单线态
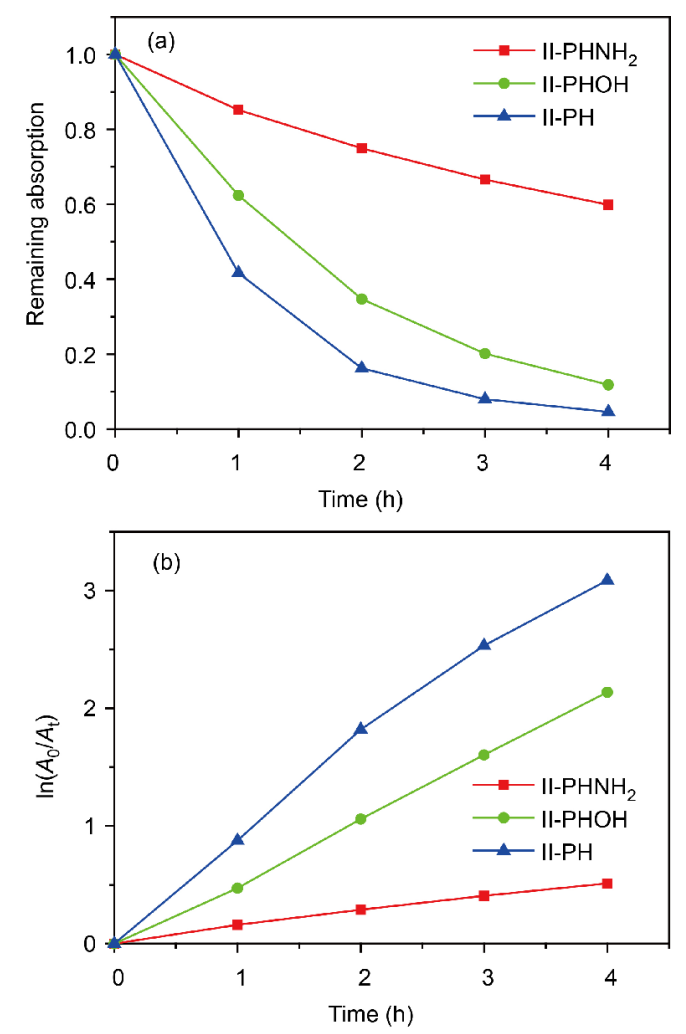

图 5 染料的光稳定性测试结果, 溶剂为水. (a) 染料残余吸 收/初始吸收 $\left(A_{\mathrm{t}} / A_{0}\right)$ 与光照时间的关系; (b) 染料 $\ln \left(A_{0} / A_{\mathrm{t}}\right)$ 与光 照时间的关系(网络版彩图)

1040
氧的进攻氧化造成的. 而单线态性氧往往产生于染料 的激发态. 分子激发态寿命短, 可降低单线态氧的生 成. 因此, 根据飞秒瞬态吸收测试系统, 测得 3 种染料 在DMSO和水中的瞬态吸收和动力学图谱, 并对其激 发态寿命进行拟合, 结果如图6所示.

从表 2 中可以看出, 3 种染料在 DMSO 中的激发 态寿命长短顺序为: II-PH (619.5 ps)>II-PHOH (525.2 ps) $>$ III-PHNH 2 (19.8 ps), 在水中激发态寿命也保持相 同的长短顺序, 与染料光降解速率一致, 表明染料激 发态寿命越短, 光稳定性越好. 同时从苂光量子产率 可以看出, 对氨基苯取代之后的菁染料荧光量子产率 最低, 说明其激发态通过辐射跃迁回到基态过程所占 比重最少, 主要通过非辐射跃迁, 降低激发态寿命. 氨 基引入, 可与菁染料母体构成分子内光诱导电子转移 (PET) 效应, 这是一个皮秒级过程, 缩短了染料在激发 态停留时间, 也就降低了处于激发态的染料与氧反应 的几率, 提高其光稳定性.

\section{4 细胞成像}

目前大多数线粒体定位类荧光探针是基于线粒 体负膜电位特性, 利用本身带有正电荷的苂光团或在 荧光团中引入带有正电荷基团, 实现对线粒体的染色, 其中最为常用的是三苯基膦正电荷基团 ${ }^{[19]}$. 由于菁染 料本身带一个正电荷, 猜测它会进入线粒体, 因此对 其进行了线粒体复染实验.

将II-PHNH 2 与商品化的核酸Hoechst 33342 和线 粒体染料MitoTracker Green FM在MCF-7细胞中进行 复染实验. 实验结果如图7所示, II-PHNH 2 和线粒体染 料MitoTracker Green FM能很好地复染于细胞线粒体, 其皮尔森复染系数 $R=0.97$, 说明染料 $\mathbf{I I}-\mathbf{P H N H _ { 2 }}$ 能够很 好地对活细胞的线粒体进行选择性定位染色.

\section{4 结论}

本文研究了 3 例中位取代的 Cy 5 菁染料, 对其光稳 定性进行分析, 并研究其光稳定性与其激发态寿命之 间的关系, 发现中位氨基苯取代的菁染料大大提升其 光稳定性, 主要是由于氨基与菁染料母体形成分子内 PET效应, 降低了其激发态寿命, 从而提高其光稳定 性. 同时发现, 此类菁染料能定位细胞线粒体, 这对细 胞线粒体长时间成像研究具有潜在的应用价值. 

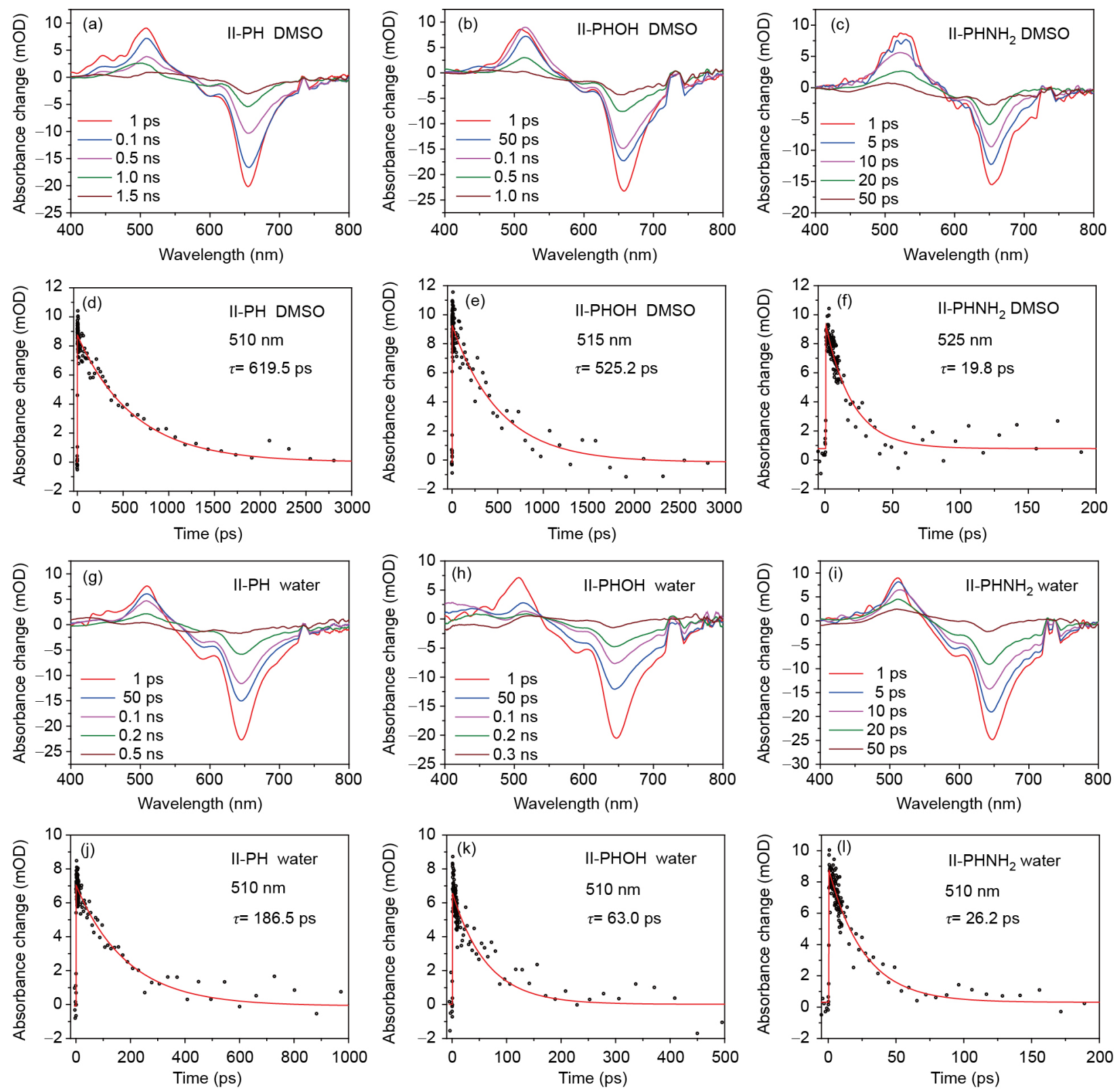

图 6 染料在DMSO和水中的飞秒时间分辨瞬态吸收光谱, $\lambda_{\mathrm{ex}}=600 \mathrm{~nm}$ 及其对应波长处的激发态吸收动力学衰减曲线(网络 版彩图)

表 2 染料在DMSO和水中对应的激发态寿命和其荧光量子产率

\begin{tabular}{ccccc}
\hline Dyes & $\tau(\mathrm{ps})$ DMSO & $\tau(\mathrm{ps})$ 水 & $\Phi_{\mathrm{f}}$ DMSO & $\Phi_{\mathrm{f}}$ 水 \\
\hline II-PHNH 2 & 19.8 & 26.2 & 0.009 & 0.008 \\
II-PHOH & 525.2 & 63.0 & 0.129 & 0.028 \\
II-PH & 619.5 & 186.5 & 0.157 & 0.038 \\
\hline
\end{tabular}



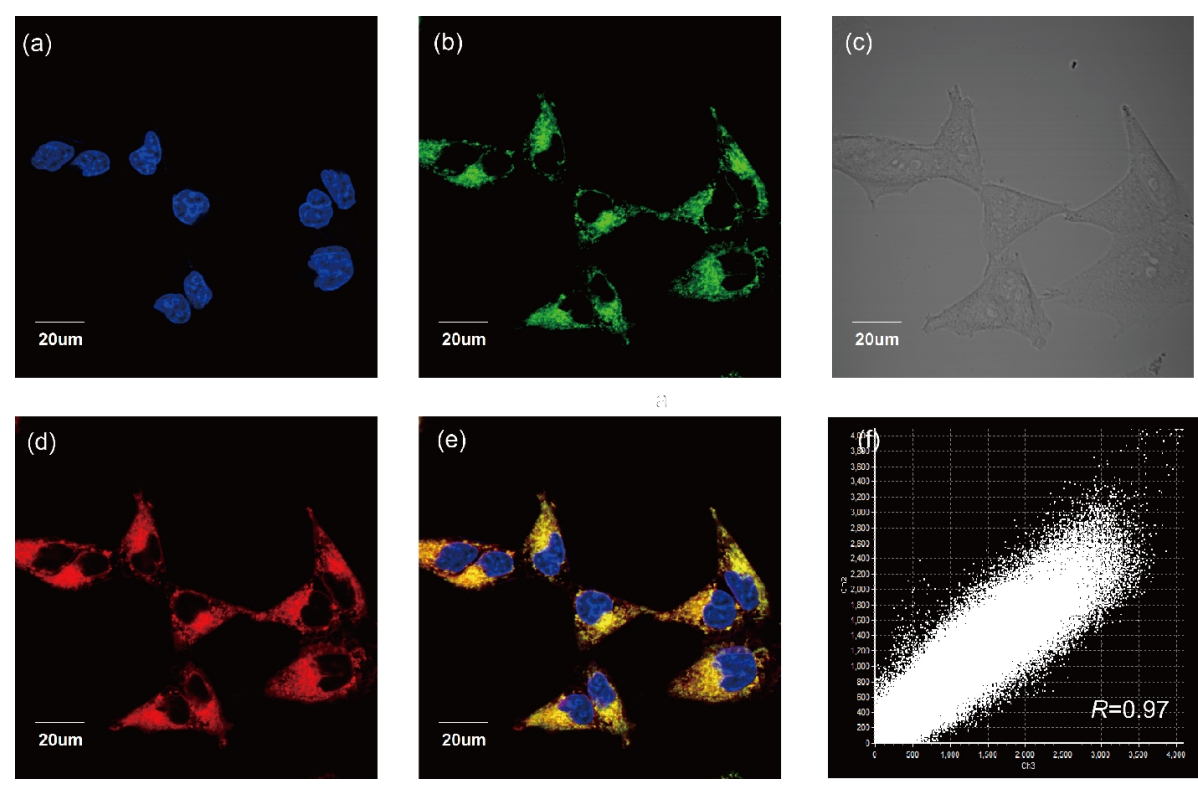

图 7 染料II-PHNH $2(1.0 \mu \mathrm{mol} / \mathrm{L})$ 、商品化线粒体染料MitoTracker Green FM $(0.2 \mu \mathrm{mol} / \mathrm{L})$ 和核酸Hoechst $33342(2.0 \mu \mathrm{g} / \mathrm{mL})$ 在 MCF-7细胞中的激光共聚焦成像图. (a) Hoechst 33342 染色 10 min; (b) MitoTracker Green FM染色 30 min; (c) 明场图; (d) 染料 II-PHNH 2 染色 $30 \mathrm{~min}$; (e) (a) (c)的叠加图; (f) 共区域化分析. 标尺为 $20 \mu \mathrm{m}$. 商业化线粒体染料MitoTracker Green FM的激发 波长为 $488 \mathrm{~nm}$, 接收波段为 $500 \sim 530 \mathrm{~nm}$; 商业化染料Hoechst 33342 的激发波长为 $405 \mathrm{~nm}$, 接受波段为 $440 \sim 480 \mathrm{~nm}$; 染料的激发 波长为 $635 \mathrm{~nm}$, 接收波段为655 755 nm (网络版彩图)

\section{参考文献}

1 Williams CG. Trans R Soc Edinb, 1857, 21: 377-401

2 张鹏超, 王升, 吴品, 汤昆, 张付利. 化学通报, 2016, 5: 455-458

3 Lv W, Chen XD, Aumiler D, Xia AD. Sci China Ser B-Chem, 2009, 52: 1148-1153

4 Yuan L, Lin W, Zheng K, He L, Huang W. Chem Soc Rev, 2013, 42: 622-661

5 李春兰, 王兰英, 孙国峰, 张祖训. 有机化学, 2006, 4: 442-453

6 Schuler B, Pannell LK. Bioconjugate Chem, 2002, 13: 1039-1043

7 Horiuchi H, Ishibashi S, Tobita S, Uchida M, Sato M, Toriba K, Otaguro K, Hiratsuka H. J Phys Chem B, 2003, 107: 7739-7746

8 曾万学, 陈萍, 盛丽琴, 张理, 郑德水, 冈崎庸树, 速水正明. 中国科学: 化学, 1995, 6: 579-584

9 Pascal S, Haefele A, Monnereau C, Charaf-Eddin A, Jacquemin D, Le Guennic B, Andraud C, Maury O. J Phys Chem A, 2014, 118: 4038-4047

10 Ornelas C, Lodescar R, Durandin A, Canary JW, Pennell R, Liebes LF, Weck M. Chem Eur J, 2011, 17: 3619-3629

11 Renikuntla BR, Rose HC, Eldo J, Waggoner AS, Armitage BA. Org Lett, 2004, 6: 909-912

12 Kim SH, Hwang SH, Kim NK, Kim JW, Yoon CM, Keum SR. Color Technol, 2000, 116: 126-131

13 Song B, Zhang Q, Ma WH, Peng XJ, Fu XM, Wang BS. Dyes Pigments, 2009, 82: 396-400

14 Nanjunda R, Owens EA, Mickelson L, Alyabyev S, Kilpatrick N, Wang S, Henary M, Wilson WD. Bioorg Med Chem, 2012, 20: 7002-7011

15 Miltsov S, Karavan V, Boyarsky V, Gómez-de Pedro S, Alonso-Chamarro J, Puyol M. Tetrahedron Lett, 2013, 54: 1202-1204

16 Wycisk V, Pauli J, Welker P, Justies A, Resch-Genger U, Haag R, Licha K. Bioconjugate Chem, 2015, 26: 773-781

17 朱良渏. 分析仪器手册. 北京: 化学工业出版社, 1997

18 王丽. 生物苂光标识用菁染料的研究. 博士学位论文. 大连: 大连理工大学, 2010. 70-72

19 姜娜, 樊江莉, 杨洪宝, 彭孝军. 化工学报, 2016, 1: 176-190 


\title{
Pentamethine cyanine located in mitochondria: relationship between photostability and excited state lifetime
}

\author{
Haiqiao Huang, Saran Long, Mingle Li, Jianjun Du, Jiangli Fan, Xiaojun Peng* \\ State Key Laboratory of Fine Chemicals, Dalian University of Technology, Dalian 116024, China \\ *Corresponding author (email: pengxj@dlut.edu.cn)
}

\begin{abstract}
Pentamethine dyes are often used for near infrared fluorescent probes owing to the advantage of high sensitivity, however, their photostability is not so satisfying. In this paper, a series of pentamethine dyes which contain electron-donating groups like amino or hydroxyl groups were synthesized, and the effect of substituents on photostability and the application in cell imaging were also discussed. The results indicated that the photostability of fluorescent dyes which possess electron donors of amino and hydroxyl groups was high, and the lifetime of excited state of fluorescent probes were reduced after leading into the amino group proved by femtosecond transient absorption, respectively. Photostability is negatively correlated with the lifetime of the excited state and the fluorescent dyes are mainly localized in mitochondria of living cells. This research will be of importance for designing cyanine dyes with high photostability and good cellular stain properties.
\end{abstract}

Keywords: cyanine dye, photostability, lifetime of excited state, bioimaging, mitochondrial

doi: $10.1360 / \mathrm{N} 032017-00061$ 